\title{
Abcès post-traumatique du psoas compliqué d'une hydronéphrose: à propos d'un cas et revue de littérature
}

\section{Post-traumatic abscess of psoas complicated by hydronephrosis: a case report and literature review}

Ouattara $\mathrm{Y}^{1}$, Koné $\mathrm{J}^{2}$, Tabue $\mathrm{L}^{3}$, Ouattara $\mathrm{Z}^{3}$, Coulibaly $\mathrm{Y}^{2}$, Traoré $\mathrm{M}^{4}$.

1. Service d'Urologie- CSREF Commune V de Bamako

2. Service d'Anesthésie- Réanimation CSREF Commune V de Bamako

3. Service d'Urologie -CHU Gabriel Touré Bamako

4. URFOSAME (Unité de recherche et de formation en santé de la mère et de l'enfant) Bamako, Université de Bamako

Auteur correspondant: Docteur Joseph Koné- drjosephkone@ yahoo.fr

\section{Résumé :}

L'abcès du psoas est une entité clinique relativement rare, en général secondaire à un foyer infectieux de voisinage (gastro-intestinal, génitourinaire ou ostéo-articulaires). L'abcès post traumatique est rare. La clinique est complexe en l'absence de tableau clinique typique. Le diagnostique est basé sur la clinique et les données radiologiques. Le traitement associe le drainage percutané et l'antibiothérapie. Nous rapportons un cas d'abcès post traumatique du psoas compliqué d'hydronéphrose et traité par abord chirurgical.

Mots Clés : Abcès du psoas, traumatisme, chirurgie Summary:

Iliopsoas abscess is a quite rare clinical entity. The Iliopsoas abscess may be secondary following gastrointestinal, urinary, osteoarticular infections. Post traumatic iliopasoas abcess in uncommon with no typical clinical characteristics. Diagnostic is based on clinical and radiologic manifestations. The association of percutaneous drainage with antibiotics is the gold standard of treatment. We report a case of posttraumatic iliopsoas abcess complicated with hydronephrosis and managed with surgical drainage.

Key words: Iliopsoas abscess, traumatism, surgical drainage

\section{Introduction}

L'abcès du posas est une entité clinique rare et de diagnostic difficile. Les cas cliniques décrits dans la littérature sont en majorité dans les pays du sud [1-4]. Il s'agit d'une collection purulente retropéritonéale localisée dans le muscle posas constitué du muscle psoas, du fusiforme et de l'iliaque triangulaire. Sa description remonte à 1881 par Mynter qui présentait les psoïtis aigus et leur classification en en fonction de l'étiopathogénie [4]. Ces abcès sont classés primitifs ou secondaires selon la présence ou non d'un autre foyer [5]. L'abcès secondaire est dû à des pathologies digestives, osseuses, urologiques ou iatrogènes [1,6-7]. L'étiologie post traumatique est rarement rapportée, et pourrait être secondaire à la suppuration d'un hématome. La symptomatologie clinique n'est pas spécifique, le diagnostic est établi à l'imagerie avec une sensibilité pour la tomodensitométrie et l'imagerie par résonnance magnétique [6]. Ces complications sont thromboemboliques ou infectieuses entrainant le décès par choc septique [1,5]. L'étiologie posttraumatique et les complications locorégionales sont rarement décrites. Nous rapportons un cas d'abcès du psoas compliqué d'hydronéphrose homolatérale chez une patiente de 15 ans immunocompétente ; dans l'objectif de discuter des étiologies, complications et la prise en charge.

Observation

Il s'agissait d'une patiente de 15 ans admise pour une tuméfaction douloureuse du flanc droit dont le début remonterait à 2 semaines environ. L'évolution serait marquée par une aggravation progressive sous traitement à base d'antibiotiques non précisés pendant 10 jours, et d'une association de paracétamol-diclofenac. L'interrogatoire retrouvait une notion de traumatisme avec point d'impact lombaire sans effraction cutanée visible avec une douleur du flanc droit soulagée par la prise d'anti-inflammatoire non stéroïdien (AINS) et le décubitus latéral gauche en chien de fusil. A l'examen physique l'on retrouvait une impotence fonctionnelle du membre inférieur droit chez une patiente asthénique avec altération de l'état, des signes de déshydratation, une polypnée à 20 cycles par minute, une température à $39^{\circ} 8 \mathrm{C}$, une pâleur conjonctivale et une tuméfaction douloureuse, chaude, de consistance dure au niveau du flanc droit. L'abdomen était souple sans contracture et la mobilisation du membre inférieur droit retrouvait un psoïtis (signe de Ludloff positif). Ailleurs, les paramètres hémodynamiques étaient stables. L'échographie retrouvait dans la fosse iliaque gauche une collection échogène, homogène réalisant un aspect de sable mouvant et siégeant dans le muscle grand psoas, mesurant 115 x 88 x 76 $\mathrm{mm}$ soit un volume de $409 \mathrm{cc}$, comprimant l'uretère gauche et entrainant en amont une hydronéphrose. A la biologie, une hyperleucocytose avec globules blancs à 20.500/mm3, un taux d'hémoglobine à 
$8 \mathrm{~g} / \mathrm{dl}$; un taux de plaquettes à $112.000 / \mathrm{mm} 3$, un taux de prothrombine à $65 \%$, créatinémie à 65,83 $\mathrm{mmol} / \mathrm{l}$. La prise en charge a consisté à un équilibre hydro électrolytique, une antibiothérapie par ceftriazone 2 grammes/24 heures et ciprofloxacine $500 \mathrm{mg} / 12$ heures, du paracétamol $1000 \mathrm{mg}$ en perfusion toutes les 06 heures et un drainage chirurgical. Cette chirurgie a été effectuée sous anesthésie générale en position de lombotomie droite et a permis l'évacuation de $600 \mathrm{ml}$ de pus franc, suivi de la pose d'un drain. L'examen bactériologique du prélèvement per-opératoire est revenu stérile. Les suites postopératoires ont été simples avec une ablation du drain à $\mathrm{J}_{12}$ post opératoire. Un contrôle réalisé à trois mois, n'a objectivé aucune anomalie clinique, radiologique ou biologique chez notre patiente.

\section{Discussion}

L'abcès du psoas est une entité clinique qui demeure complexe sur le plan étiologique et diagnostique. De nombreuses séries ont été rapportées dans la littérature, cependant l'épidémiologie reste difficile à établir du fait de la difficulté du diagnostique et thérapeutique [8-9]. Il est le plus souvent secondaire; des formes primitives sont observées chez des terrains fragiles (enfants, immunodéprimés) [9-12]. Ces abcès secondaires font suite à des pathologies digestives, viscérales ou ostéo-articulaires telles que la maladie de Crohn, l'appendicite, la diverticulite, le cancer $\mathrm{du}$ colon, les spondylodiscites tuberculeuses, les arthrites sacro-iliaques et les arthrites de hanche, les pyélonéphroses $[\mathbf{2 , 3}, \mathbf{5}]$. L'infection musculaire survient par voie hématogène ou par contigüité à partir d'un site voisin infecté. Des causes iatrogènes ont été décrites telles que l'abord veineux central, les blocs nerveux périphériques $[\mathbf{7 , 1 3}$. L'hypothèse de surinfection d'un hématome post traumatique expliquerait le cas de notre patiente. Dans notre cas, le contexte clinique était évocateur, et la recherche d'un site primitif négative. La triade symptomatique composée de douleur abdominolombaire, psoïtis et empâtement de la fosse iliaque, en fait classiquement le diagnostic positif. L'imagerie est d'une contribution considérable dans le diagnostic $[\mathbf{1 4}, \mathbf{1 5}]$. L'échographie semble moins sensible que la tomodensitométrie [16]. Comme ce fût le cas dans notre cas, l'échographie a contribué au diagnostic. L'apport de l'imagerie a un double intérêt diagnostique et thérapeutique (drainage échoguidé ou scannoguidé) [6,15]. Le diagnostic différentiel de cette affection se pose avec le plastron appendiculaire, la lymphadénite inguinale, l'abcès pariétal, l'abcès para-néphrétique, les tumeurs du compartiment du muscle psoas, le kyste hydatique du psoas ou l'hématome du psoas. Le drainage percutané constitue actuellement le traitement le plus courant et convient mieux aux patients généralement débilités. Il s'agit d'un traitement fiable et peu invasif $[\mathbf{8 , 1 5}$. Le recours à la chirurgie est peu fréquent environ $8 \%$ des cas. Il serait beaucoup plus indiqué dans les abcès secondaires, les abcès multiloculaires et dans les cas où le drainage percutané s'est avéré insuffisant $[1,9,17]$.Le décès survient en général par choc septique ou dans un tableau d'embolie pulmonaire [1]. D'autres complications ont été rapportées comme que la compression des voies urinaires; comme ce fût le cas chez notre patiente. Dans la recherche étiologique d'une hydronéphrose unilatérale, l'abcès du psoas pourrait bien être une étiologie à évoquer [8]. Le pronostic dépend de la rapidité du traitement et de son caractère adapté [1]. Conclusion

L'abcès post traumatique du psoas est une entité clinique rare, pouvant avoir des complications locales comme l'hydronéphrose par compression de l'urétérale. Le diagnostic repose dans un contexte post-traumatique sur la clinique et l'imagerie (échographie et tomodensitométrie). Le traitement consiste au drainage de l'abcès, avec une évolution favorable.

\section{Conflit d'intérêt : Aucun \\ Références}

1. Conde RC, Estebanez ZJ, Rodrigues TA, Amon SJ, Simal F, Martinez SJM. Traitement de l'abcès du psoas : drainage percutané ou chirurgie ouverte. Prog Urol 2000; 10:418-23

2. Laguna P, Moya M. Abscess of the psoas muscle: analysis of 11 cases and review of the literature. Enferm Infecc Microbiol Clin 1998; 16 (1):19-24.

3. Audia S, Martha B, Grappin M, Duong M, Buisson M, Couaillier JF, et al. Les abcès pyogènes secondaires du psoas: à propos de six cas et revue de la littérature. Rev Med Interne 2006; 27:828-35

4. Mynter H. Acute psoitis. Buffalo Med Surg J 1881; 21:202-10.

5. Leclercq P, Loly C. Spondylodiscite compliquée d'un abcès du psoas. Rev Med Suisse 2007; 101(3):2492

6. Garcia E, Gutierrez J, Diaz CM. Abscesos del psoas: presentación de ocho casos y revisión de la literatura. Rev Clin Esp 1995; 195:289-93.

7. Adam F, Jaziri S, Chauvin M. Psoas abscess complicating femoral nerve block catheter. Anesthesiology 2003; 99:230-1.

8. Asseban M, Qarro A, Bazine K, Beddouch A, Lezrek M, Ammani A, Alami M. Drainage percutané de l'abcès du psoas: Notre expérience et revue de la littérature. Afr J Urol 2014; 20 (1):4247.

9. Garner JP, Meiring PD, Ravi K, Gupta R. Psoas abscess - not as rare as we think? Colorectal Dis 2007 ; 9(3) :269-74.

10. Al-Zaiem MM, Bajuifer SJ, Fattani MO, AlZaiem FM. Bilateral iliopsoas abscess associated with right hip septic arthritis in a neonate (Article). Saudi Med J 2014; 35(7):743-6. 
11. Patel R, Pimpalwar A, Hutton K. Primary neonatal iliopsoas abscess. J Ped Surg Case Reports 2013; 1(2):11-13.

12. Taiwo B. Psoas abcess: a primer for the internist. South Med J 2001; 94:2-5

13. Moran KM, Finkbeiner AA. Iliopsoas abscess following catheterization of the femoral artery: diagnostic and treatment strategies. Am J Orthop 1997; 26(6):446-8.

15. Moudouni MS, Dahami Z, Hocar O, Gabsi M, Boukhari M, Barjani F, ELhaous A, Lakmichi MA, Sark I. Les abcès du psoas: aspects diagnostic et thérapeutique. Afr J Urol 2005; 11(4):319-22

15. Cantasdemir M, Kara B, Cebi D, Selcuk ND, Numan F. Computed tomography-guided percutaneous catheter drainage of primary and secondary iliopsoas abscesses. Clin Radiol 2003; 58:811-5

16. Paley M, Sidhu PS, Evans RA, Karani JB. Retroperitoneal collections-aetiology and radiological implications. Clin Radiol 1997; 52:290-4

17. Charalampopoulos A, Macheras A, Charalabopoulos A, Fotiadis C, Charalabopoulos K. Iliopsoas abscesses: diagnostic, aetiologic and therapeutic approach in five patients with a literature review. Scand J Gastroenterol 2009; 44(5):594-9

18. Finnerty RU, Vordermark JS, Modarelli RO, Buck AS. Primary psoas abscess: case report and review of the Literature. J Urol 1981; 126(1):108-9. 